\title{
Pancreatic perfusion and subsequent response to glucose in healthy individuals and patients with type 1 diabetes
}

\author{
Lina Carlbom $^{1}$ • Daniel Espes ${ }^{2,3}$ • Mark Lubberink ${ }^{1}$ • Olof Eriksson ${ }^{4} \cdot$ Lars Johansson $^{1}$ • \\ Leif Jansson $^{2}$ • Olle Korsgren ${ }^{5}$ - Håkan Ahlström ${ }^{1}$ • Per-Ola Carlsson ${ }^{2,3}$
}

Received: 11 January 2016 / Accepted: 23 May 2016/Published online: 16 June 2016

(C) Springer-Verlag Berlin Heidelberg 2016

\begin{abstract}
Aims/hypothesis The aim of this study was to investigate pancreatic perfusion and its response to a glucose load in patients with type 1 diabetes mellitus compared with non-diabetic ('healthy') individuals.

Methods Eight individuals with longstanding type 1 diabetes and ten sex-, age- and BMI-matched healthy controls underwent dynamic positron emission tomography scanning with ${ }^{15} \mathrm{O}-\mathrm{la}-$ belled water before and after intravenous administration of glucose. Perfusion in the pancreas was measured. Portal and arterial hepatic perfusion were recorded as references.

Results Under fasting conditions, total pancreatic perfusion was on average $23 \%$ lower in the individuals with diabetes compared with healthy individuals. Glucose increased total pancreatic and portal hepatic blood perfusion in healthy individuals by $48 \%$ and $38 \%$, respectively. In individuals with diabetes there was no significant increase in either total pancreatic or portal hepatic perfusion.
\end{abstract}

Olle Korsgren, Håkan Ahlström and Per-Ola Carlsson contributed equally to this study.

\section{Per-Ola Carlsson}

per-ola.carlsson@mcb.uu.se

1 Department of Surgical Sciences, Radiology, Uppsala University, Uppsala, Sweden

2 Department of Medical Cell Biology, Uppsala University, Husargatan 3, SE-751 23 Uppsala, Sweden

3 Department of Medical Sciences, Uppsala University, Uppsala, Sweden

4 Department of Medicinal Chemistry, Preclinical PET Platform, Uppsala University, Uppsala, Sweden

5 Department of Immunology, Genetics and Pathology, Uppsala University, Uppsala, Sweden
Conclusions/interpretation Individuals with type 1 diabetes have reduced basal pancreatic perfusion and a severely impaired pancreatic and splanchnic perfusion response to intravenous glucose stimulation.

Keywords Blood flow · Glucose $\cdot$ Pancreas · Pancreatic islets · Perfusion · Type 1 diabetes
Abbreviations
CT Computed tomography
PET Positron emission tomography
ROI Regions of interest
VOI Volumes of interest

\section{Introduction}

Positron emission tomography (PET) with radioactive labelled water $\left(\left[{ }^{15} \mathrm{O}\right] \mathrm{H}_{2} \mathrm{O}\right)$ as a tracer is a well-established tool to study changes in perfusion in human organs and has previously been used to study pancreatic perfusion under basal conditions $[1,2]$. Pancreatic perfusion is normally closely related to tissue function, for example secretin, a hormone which stimulates exocrine secretion, also increases pancreatic perfusion in humans [3]. The perfusion difference between insulin-deficient and healthy animals mainly reflects the islet vascular component, i.e. the islet blood flow contribution [4-6]. Moreover, a 2-3 fold increase in islet blood flow after intravenous glucose administration, which facilitates the release and dispersal of insulin, is consistently seen in healthy rodents $[4,5]$. In this study we set out to study pancreatic perfusion under basal conditions and its responsiveness to glucose in non-diabetic ('healthy') and type 1 diabetic individuals. 


\section{Methods}

All procedures were approved by the regional ethical board and performed in accordance with the Helsinki declaration. Written informed consent was obtained from all participants prior to their inclusion.

Inclusion criteria for the type 1 diabetes participants were: $18-35$ years, $>10$ years diabetes duration, $\mathrm{HbA}_{1 \mathrm{c}}<7.5 \%$ ( $58.5 \mathrm{mmol} / \mathrm{mol}$ ), fasting C-peptide $<0.05 \mathrm{nmol} / \mathrm{l}$, no medication besides insulin, plasma creatinine $<90 \mu \mathrm{mol} / \mathrm{l}$, urine albu$\min <25 \mathrm{mg} / \mathrm{l}$ and no signs of retinopathy or known other micro- or macrovascular complication. Age-, sex- and BMImatched healthy individuals were recruited by advertising. All study participants were non-smokers and physically active. One of the diabetic participants had a very small pancreas, with substantial spill over of PET-signal from the left kidney into the pancreatic regions of interest (ROI) and was, therefore, excluded. See Table 1 for descriptive data of included participants.

All participants were fasting for $>4 \mathrm{~h}$ prior to the PET examinations. Eight of the ten healthy individuals and all (eight) participants with diabetes underwent one $10 \mathrm{~min}$ baseline and one $10 \min \left[{ }^{15} \mathrm{O}\right] \mathrm{H}_{2} \mathrm{O}$ dynamic PET examination (400 MBq each) directly after an intravenous injection of $30 \%$ (wt $/ \mathrm{vol}$.) glucose solution $(300 \mathrm{mg} / \mathrm{kg})$. The remaining two healthy individuals were investigated with dynamic PET prior to and 10-20 min after glucose administration.

The pancreas was positioned in the centre of the $15 \mathrm{~cm}$ axial field of view of a Discovery ST PET/computed tomography (CT) scanner (GE Healthcare, Milwaukee, MI, USA) by assistance of a low-dose CT scout view (140 kV, $10 \mathrm{mAs}$ ). Image acquisition was performed in 3D (26 frames). Images were reconstructed using an iterative Ordered Subsets
Expectation Maximisation (OSEM) VUEpoint algorithm (GE Healthcare). Data were analysed using the VOIager 4.0.7 software (GE Healthcare, Uppsala, Sweden). ROIs corresponding to the pancreas and its parts were delineated on transaxial sequential CT slices and combined into volumes of interest (VOIs). The border of the tail (cauda) of the pancreas was assigned to the medial border of the left kidney and the head (caput) of the pancreas was defined as the pancreatic region lateral to the superior mesenteric artery. In order to reduce possible partial volume effect, the ROIs were delineated slightly inside the pancreatic contour. CT VOIs were transferred to co-registered $\left[{ }^{15} \mathrm{O}\right] \mathrm{H}_{2} \mathrm{O}-\mathrm{PET}$ images. Perfusion was assessed by a standard single-tissue compartment model from dynamic pancreatic $\left[{ }^{15} \mathrm{O}_{\mathrm{H}} \mathrm{O}-\mathrm{PET}\right.$ data, using an aortic VOI as the input function [1].

Portal and arterial hepatic perfusion were calculated and used as comparators [1]. The data of one healthy individual could not be fitted accurately to this model and was therefore excluded.

Statistical analysis Differences between two groups of parametric data were assessed by two-sided $t$ tests. Normal distribution of data was assessed by Shapiro-Wilk's test and equal variance was assessed by Levene's test. Pearson's Product Moment Correlation Coefficient was calculated to assess linear correlations. $p<0.05$ was considered statistically significant. All values are given as means \pm SEM.

\section{Results}

As expected, $\mathrm{HbA}_{1 \mathrm{c}}$ levels were increased in individuals with type 1 diabetes. However, after glucose administration, both
Table 1 Descriptive data of study participants

\begin{tabular}{lll}
\hline Variable & HI & T1D \\
\hline$n$ & 8 & 7 \\
Male $(n, \%)$ & $4(50 \%)$ & $4(57 \%)$ \\
Age (years) & $26.7 \pm 1.3$ & $25.9 \pm 0.9$ \\
BMI $\left(\mathrm{kg} / \mathrm{m}^{2}\right)$ & $22.9 \pm 1.4$ & $23.6 \pm 1.0$ \\
HbA1c \% DCCT & $5.2 \pm 0.3$ & $7.1 \pm 0.3 * * *$ \\
HbA1c (mmol/mol) & $(33.3 \pm 0.82)$ & $(53.6 \pm 1.3)^{* * *}$ \\
fP-glucose (mmol/l) & $5.4 \pm 0.2$ & $6.7 \pm 1.0$ \\
P-glucose (mmol/l) following glucose administration & $12.1 \pm 0.3$ & $14.3 \pm 1.01$ \\
fP-C-peptide (nmol/l) & $0.6 \pm 0.07$ & $0.01 \pm 0.006^{* * *}$ \\
C-peptide (nmol/l) following glucose administration & $1.6 \pm 0.3$ & $0.01 \pm 0.006^{* * *}$ \\
\hline
\end{tabular}

Five individuals with type 1 diabetes had non-detectable C-peptide levels and were therefore assigned the lowest detectable level for the assay $(0.003 \mathrm{nmol} / \mathrm{l})$

All values are given as means \pm SEM

Unpaired Student's $t$ test was used to determine differences between the two groups

$p$ values $<0.05$ were considered statistically significant. $* * *$ denotes $p<0.001$

$\mathrm{fP}$, fasting plasma, HI, healthy individuals; P-glucose, plasma glucose; T1D, type 1 diabetes 
mean fasting glucose and stimulated glucose concentrations were similar to healthy individuals during the PET examination (Table 1).

Participants with type 1 diabetes had a $23 \%$ lower baseline pancreatic perfusion compared with healthy participants $\left(1.3 \pm 0.1\right.$ vs $1.7 \pm 0.1 \mathrm{ml} \mathrm{x} \mathrm{min}^{-1} \mathrm{x} \mathrm{g}^{-1} ; p=0.0497$; Fig. 1a). A lower perfusion seemed consistent in all parts of the pancreas of type 1 diabetic individuals, although when compared with the findings from healthy participants, this change was only statistically significant for the pancreatic head (caput; $p=0.0298$ ) (Fig. 1a,b). There was no difference in either portal or arterial hepatic perfusion between healthy and type 1 diabetic participants (Fig. 1a). In the healthy individuals only, an inverse correlation was observed between baseline pancreatic blood flow and plasma glucose concentrations (Fig. 1c,d).

In healthy individuals, a $48 \pm 8 \%$ increase $(p=0.0006$, compared with baseline measurements) in total pancreatic perfusion was observed during the first $10 \mathrm{~min}$ after intravenous glucose administration (the change in absolute values is shown in Fig. 1e,g) and similar increases were observed in all subregions of the pancreas (the change in absolute values is shown in Fig. 1e-h). The pancreatic perfusion increase in response to glucose was much less pronounced in type 1 diabetic participants irrespective of their fasting glucose levels (the change in absolute values is shown in Fig. 1e,g). There was only a mean $10-12 \%$ increase when compared with baseline measurements in the pancreatic head (caput; $p=0.048$ ) and tail (cauda; $p=0.004)$, but no statistically significant change in total pancreatic perfusion $(p=0.065)$ in these individuals. Neither portal nor arterial hepatic perfusion increased in diabetic individuals after intravenous glucose injection, while there was a $38 \pm 10 \%(p=0.0083)$ increase when compared with baseline measurements in the portal, but not arterial hepatic, perfusion in healthy individuals (the change in absolute values is shown in Fig. 1g). There was no correlation between change in plasma insulin concentrations and pancreatic blood flow in healthy individuals after glucose administration (Fig. 1i-j). Additionally, the magnitude of pancreatic and portal hepatic perfusion increase in healthy individuals did not show any correlation (Fig. 1k). In the two healthy individuals re-examined 10-20 min after glucose administration, there was no longer any pancreatic blood flow increase $(1.6 \pm 0.3$ vs 1.7 $\left.\pm 0.3 \mathrm{ml} \times \min ^{-1} \times \mathrm{g}^{-1}\right)$, despite blood glucose concentrations remaining increased $20 \mathrm{~min}$ after glucose administration $(11.3 \pm 0.2 \mathrm{mmol} / \mathrm{l})$ (data not shown).

\section{Discussion}

We report a lower baseline perfusion of the pancreas in individuals with long-standing type 1 diabetes when compared with healthy individuals. In previous investigations, pancreatic perfusion in individuals with type 1 diabetes has been shown either not to change or to have a tendency to decrease $[2,7]$. Experience from animal studies [4-6] is suggestive that the perfusion difference between insulin-deficient and healthy animals mainly reflects the islet vascular component. Direct studies of islet blood flow in humans cannot be performed, since the techniques currently available for use in animal studies are invasive and/or terminal $[4,5]$. Pancreatic islets are dependent on the effective transport of oxygen to the tissue for glucose metabolism, and a high perfusion for their dispersal of insulin $[5,8]$. It is of interest to note that in the healthy individuals an inverse relation between fasting plasma glucose concentrations and pancreatic perfusion was observed, suggesting that higher perfusion may have increased insulin dispersal into the portal circulation. Importantly, in the present study pancreas perfusion was determined from the clearance rate constant of water, which is dependent on the shape of the time-activity curve rather than its amplitude. Thus, it may be assumed that the partial volume effects associated with the smaller pancreas volumes in individuals with type 1 diabetes would not have contributed to the difference in perfusion observed [9].

We found that all diabetic individuals had an impaired perfusion response to glucose, with a negligible increase in pancreatic perfusion compared with that observed in healthy individuals. An increased islet perfusion in healthy individuals would facilitate insulin dispersal into the systemic circulation, but may not necessarily be linked to an increment in glucosedependent insulin secretion [10]. Accordingly, we did not observe any correlation between the increase in plasma insulin concentrations and perfusion after glucose administration in healthy individuals.

However, although not shown to correlate on an individual basis, the increase in pancreatic perfusion after glucose infusion in healthy individuals was accompanied by an increase in portal hepatic perfusion, when compared with baseline measurements, to which the pancreatic contribution is only a minor fraction [11]. It is therefore likely that the pancreatic perfusion increase observed in this study did not only reflect an islet blood flow increase, as seen in rodents [4, 5], but may also have occurred as a result of general blood flow increase, caused by parasympathetic signalling through the vagal nerve [12]. In line with this, the absence of an increase in pancreatic perfusion in diabetic individuals may be explained by autonomic neuropathy or endothelial dysfunction [13], albeit the type 1 diabetic participants involved in this study were chosen based on good metabolic control and absence of known microvascular complications. Additionally, the lack of an increase in arterial hepatic perfusion could be explained by the hepatic arterial buffer response to increased portal flow. Future studies in large animals may be of value to increase our understanding of the partial contribution of 

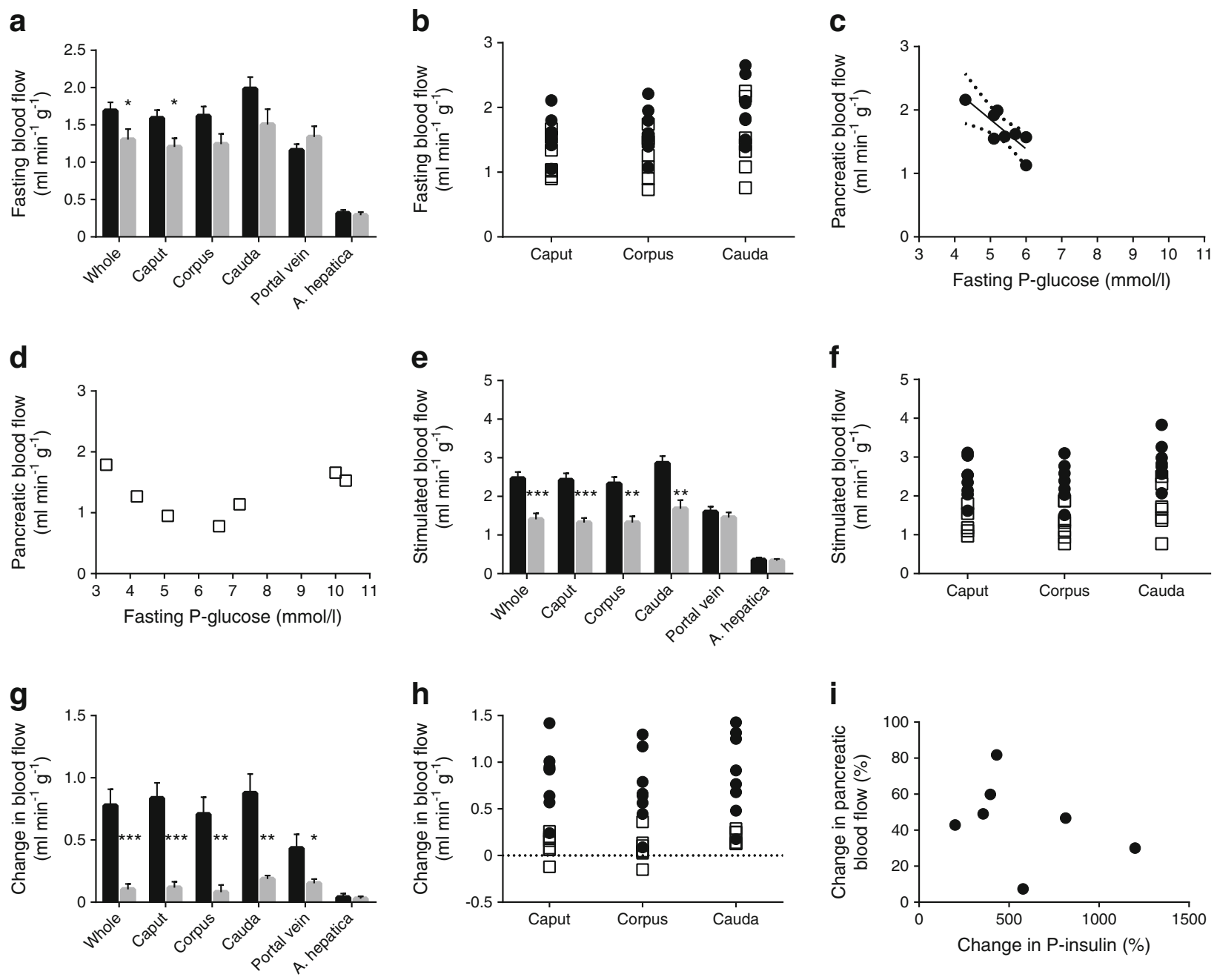

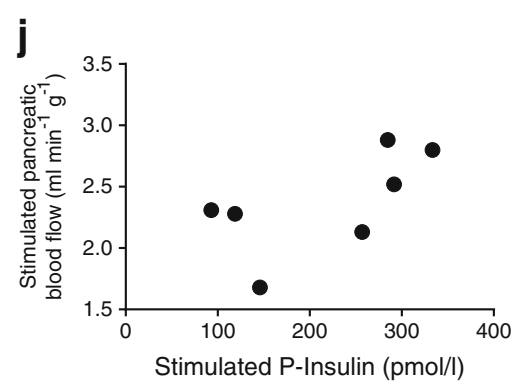

Fig. 1 Blood perfusion of the pancreas and its anatomical subregions (head [caput], body [corpus] and tail [cauda]) and in liver tissue (portal vein and hepatic artery [A. hepatica]) in healthy individuals $(n=8)$ and individuals with long-standing, well-controlled type 1 diabetes $(n=7)$ under baseline and glucose stimulated conditions. (a) Baseline perfusion in the whole pancreas, anatomical subregions of the pancreas, and in the liver tissue of healthy individuals and individuals with type 1 diabetes. (b) Individual blood flow values for the anatomical subregions for healthy and type 1 diabetic study participants. (c, d) Analysis of the correlation between fasting plasma glucose (P-glucose) concentrations and baseline pancreatic blood flow in (c) healthy $\left(p=0.014, r^{2}=0.66\right)$ and (d) type 1 diabetic individuals $\left(p=0.74, r^{2}=0.024\right)$. (e) Effects of intravenous glucose administration on perfusion of the whole pancreas and its anatomical subregions and liver tissue. (f) Glucose-stimulated blood flow in the anatomical subregions of the pancreas for each study participant. (g) The

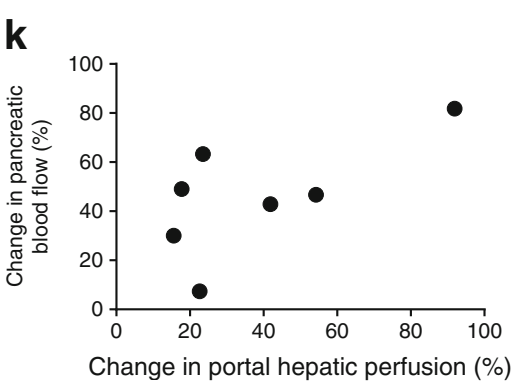

change in blood flow in response to intravenous glucose administration in the whole pancreas, anatomical subregions of the pancreas and liver tissue. (h) The change in blood flow in the anatomical subregions of the pancreas in response to intravenous glucose administration for each study participant. (i) Analysis of correlation between change in plasma insulin (P-insulin) and pancreatic blood flow after glucose administration in healthy individuals $\left(p=0.44, r^{2}=0.13\right)$ and in $(\mathbf{j})$ as absolute values $\left(p=0.12, r^{2}=041\right)$, expressed as per cent increase. (k) Analysis of correlation between changes in portal hepatic perfusion and pancreatic blood flow in healthy individuals, expressed as per cent increase $(p=0.24$, $\left.r^{2}=0.29\right)$. All values in (a), (e) and (g) are given as means \pm SEM. Healthy individuals, black bars or black circles; individuals with type 1 diabetes; grey bars or white squares. ${ }^{*} p<0.05, * * p<0.01$ and $* * * p<0.001$ when compared with healthy individuals 
islet blood flow increase and general increase in blood perfusion of splanchnic organs.

In contrast to our results, a previous study of human pancreatic blood flow did not show any difference between individuals with type 1 diabetes and healthy individuals, neither at baseline nor after glucose administration [7]. This disparity in findings could be due to the limitations of the technique that was used; arterial spin labelling MRI is a technique that has a high noise to signal ratio, where artefacts are induced by surrounding organ movements and where there are an insufficient number of voxels for sensitive analysis. In comparison, the technique used in the present study demonstrates the possibility of assessing both inter-individual differences and dynamic changes in pancreatic perfusion in a small sample size. This study, therefore, provides evidence for a potentially valuable tool for further studies of pancreatic perfusion in diabetes to gain additional understanding of the possible consequences of dysfunctional blood flow regulation as part of the disease.

Funding This study received financial support from the Swedish Research Council (K2013-55-X-15043-10-5; K2015-54X-12219-19-4; K2013-64X-08268-26-3), the Swedish Diabetes Foundation, the Swedish Juvenile Diabetes Foundation, Diabetes Wellness Sverige, The European Foundation for the Study of Diabetes (EFSD)/Novo Nordisk and the Novo Nordisk Foundation.

Duality of interest The authors declare that there is no duality of interest associated with this manuscript.

Contribution statement All authors conceived and designed the study and participated in the analysis and interpretation of the data. LC drafted the manuscript and the other authors revised it critically for intellectual content. All authors approved the final version of the paper. POC is the guarantor of this work.

\section{References}

1. Honka H, Hannukainen JC, Tarkia M et al (2014) Pancreatic metabolism, blood flow, and beta-cell function in obese humans. J Clin Endocrinol Metab 99:E981-990

2. Eriksson O, Espes D, Selvaraju RK et al (2014) Positron emission tomography ligand [11C]5-hydroxy-tryptophan can be used as a surrogate marker for the human endocrine pancreas. Diabetes 63:3428-3437

3. Cox EF, Smith JK, Chowdhury AH, Lobo DN, Francis ST, Simpson J (2015) Temporal assessment of pancreatic blood flow and perfusion following secretin stimulation using noninvasive MRI. J Magn Reson Imaging 42:1233-1240

4. Nyman LR, Ford E, Powers AC, Piston DW (2010) Glucosedependent blood flow dynamics in murine pancreatic islets in vivo. Am J Physiol Endocrinol Metab 298:E807-814

5. Jansson L, Hellerstrom C (1983) Stimulation by glucose of the blood flow to the pancreatic islets of the rat. Diabetologia 25:45-50

6. Lifson N, Kramlinger KG, Mayrand RR, Lender EJ (1980) Blood flow to the rabbit pancreas with special reference to the islets of Langerhans. Gastroenterology 79:466-473

7. Hirshberg B, Qiu M, Cali AM et al (2009) Pancreatic perfusion of healthy individuals and type 1 diabetic patients as assessed by magnetic resonance perfusion imaging. Diabetologia 52:1561-1565

8. Dionne KE, Colton CK, Yarmush ML (1993) Effect of hypoxia on insulin secretion by isolated rat and canine islets of Langerhans. Diabetes 42:12-21

9. Lubberink M, Harms HJ, Halbmeijer R, de Haan S, Knaapen P, Lammertsma AA (2010) Low-dose quantitative myocardial blood flow imaging using 15O-water and PET without attenuation correction. J Nucl Med 51:575-580

10. Jansson L, Barbu A, Bodin B et al (2016) Pancreatic islet blood flow and its measurement. Ups J Med Sci 121:81-95

11. Ferguson JL, Merrill GF, Miller HI, Spitzer JJ (1977) Regional blood flow redistribution during early burn shock in the guinea pig. Circ Shock 4:317-326

12. Hickson JC (1970) The secretory and vascular response to nervous and hormonal stimulation in the pancreas of the pig. J Physiol 206:299-322

13. Hamilton SJ, Watts GF (2013) Endothelial dysfunction in diabetes: pathogenesis, significance, and treatment. Rev Diabet Stud 10:133-156 